\title{
La condanna della tessitura. Origine e funzione di un motivo narrativo nel Roman de Jaufre e in altri romanzi arturiani
}

\section{The Condemnation of the Tessitura: Origin and Function of a Narrative Motif in Roman de Jaufre and other Arthurian Novels}

\author{
MARgherita LeCCo \\ Università degli Studi di Genova \\ 34641@unige.it
}

\begin{abstract}
Nei romanzi medievali (ad iniziare da Jaufre, composto intorno al 1225), i cavalieri impegnati nell'aventure si trovano a volte costretti ad una prova di tessitura di stoffe. Questa prova è considerata infamante perché collegata al lavoro manuale. Solo i cavalieri più valorosi, o che possono contare su un cavaliere amico (come Lancelot aiutato da Gauvain nelle Merveilles de Rigomer, fine del secolo XIII), riescono a sottrarvisi, abbattendo coloro che li hanno costretti a questa infamia e ritrovando poi la loro libertà e il loro onore. Ma questo locus narrativo diviene presto un 'motivo' importante nell'economia narrativa dei romanzi.
\end{abstract}

Palabras clave: Jaufre, romanzi medievali, romanzi di cavalleria, tecniche di costruzione narrativa, 'motivi' letterari

In medieval novels (beginning with Jaufre, about 1225), the knights involved in the adventure are sometimes forced to a weaving punishement. This test is considered infamous because it is linked to a manual work. Only the most skilled knights or those who find a friendly knight (like Lancelot helped from Gauvain in the Merveilles de Rigomer, late XIII Century) are able to get rid of them, defeating the enemy who forced them and rediscovering their freedom and their honor. But this becomes an important narrative 'motif'.

KEYwORDS: Roman de Jaufre, medieval novels, chivalric 'aventure', mematter and structures of the medieval 'motifs' 
A 1 Roman de Jaufre si attribuiscono forse troppi padri, rintracciando nella sua composizione il convergere di molte tradizioni letterarie (Lecco, “Testo, immagine"). ${ }^{1}$ In qualche caso, però, è ancora possibile che relazioni testuali non manifeste si propongano all'attenzione: casi sporadici, per lo più, da rintracciare come occorrenze disperse e rarefatte, all'interno di una compagine testuale che ha, in gran parte, da tempo rivelato i suoi più cospicui intertesti e i legami che inseriscono il romanzo in una fitta trama sia narrativa che lirica, riconoscendo all'Autore del Jaufre numerose e proficue conoscenze, non distanti da quella competenza che Guiraut de Cabreira chiedeva al suo Cabra joglar. ${ }^{2}$ Uno di questi casi si rintraccia, come è forse possibile sostenere, in relazione ad un passo che attirerebbe comunque l'attenzione per ragioni relative all'interpretazione del romanzo.

$\mathrm{Al}$ v. 1362 ss., come si ricorderà, ${ }^{3}$ il cavaliere Jaufre, partito dalla corte del reiArtus (90) in cerca di avventure, avventure che si succedono per lui con frequenza, difficoltà e carica orrifica crescenti, s'imbatte in un albero cui è appesa una lancia: il solo sfiorarla provoca la comparsa di un nano, poi di un cavaliere, che sfida Jaufre a duello. È infatti proibito cercare di impadronirsi della lancia, che è fatata, come indica il suo colore bianco oltremondano (lança qu'es tota blanca, 1368): come spiega il cavaliere, coloro che tentano l'impresa sono condannati ad una sfida, perduta la quale, se sconfitti, vengono inesorabilmente impiccati (1447); l'unica via per sottrarsi alla disfatta comporta una ricusazione del duello, e l'accettazione di alcune condizioni. Jaufre, prima di gettarsi nell'impresa, si informa su tali condizioni e si sente rispondere che esse consistono nella rinuncia ad un certo numero di pratiche molto comuni:

$$
\begin{aligned}
& \ll[\ldots] \text { que ja mais a sa vida } \\
& \text { Non cavalges, ni no.s tolges } \\
& \text { Cabels ni onglas que ages, } \\
& \text { Ni non manjes pan de forment, } \\
& \text { Ni begues vin, ne vestiment }
\end{aligned}
$$

\footnotetext{
${ }^{1}$ Dove si ipotizzava una relazione tra alcuni passagi del romanzo e l'Alexandre di Alexandre de Paris, sostenibile forse per la disposizione dei planhs dei vv. 8479-8734, meno per altri elementi. Per uno studio sulle fonti di Jaufre vid. specialmente Pontecorvo ("Una fonte"), Limentani ("I problemi del Jaufre"), e Lee (Jaufre, 15ss.).

${ }^{2}$ Per Cabra juglar, oltre a Pirot (Recherches, 371-378); vid. Lafont ("Relecture”).

${ }^{3}$ Per le edizioni di Jaufre: Breuer (Jaufre), Brunel (Jaufre. Roman arthurien), Lavaud-Nelli (Le Roman de Jaufre), Lee (Jaufre), e la traduzione inglese di Ross (Jaufre. An Occitan Arthurian Romance). Da Lee, che può essere considerata edizione di riferimento, sono tratte tutte le citazioni qui riportate.
} 
Non portes, si el no.l tesia.

E se neguns aicho fazia,

Enantz c'ab me se conbates,

Poiria esser, que la.i trobes»

A quest'ultima condizione, Jaufre si sente spinto a chiedere come potrà mai fare, dal momento che non sa tessere: "«E se non saps far vestimentz?»" (1465). Domanda cui il cavaliere risponde con insolenza che gliene farà insegnare le tecniche:

«Ieu t'o farai mout ricamentz,

Ditz lo cavallier, enseignar,

Teiser e coisir e taillar»

$(1466-1468)$

All'imposizione, Jaufre si schermisce, e la minaccia lo motiva infine ad accettare il duello, dal quale uscirà vincitore:

«Cho, ditz Jaufre, non farai res,

Que greu o auria apres».

$\ll$ Si faras, car fortz iest e grantz, Ainz que sion passetz.v. anz». $\ll J a$, per ma fe, ren no.n farai,

Ditz Jaufre, aintz me conbatrai»

(1471-1476)

Questo episodio del Jaufre non ha, a quanto so, mai suscitato un interesse particolare: lo si considera, al più, formato per analogia sul tipo motivale-narrativo di molte altre avventure romanzesche, che impongono prove che impegnano i cavalieri anche sotto il rispetto corporale. Può essere, invece, che l'episodio sia formato a partire da un modello particolare, e da un rimando molto ben individuabile, rintracciato il quale si potrebbe avere anche una conferma su un altro, pur lieve, fattore del testo.

\section{INTERTESTI ARTURIANI}

A voler cercare unascendenza testuale dietro le modalità della condanna espressa dal Cavaliere della Blanca Lança si dovrebbe riandare, in prima istanza, 
all'episodio delle fanciulle tessitrici dell'Yvain (Poirion et al. ed., Chrétien de Troyes, Euvres complètes, 5187-5696, sp. 5193-5348). Episodio che, per quanto introdotto con qualche tratto di coloritura bretone (i due Luiton), non possiede fondo folclorico e va rivendicato alle implicazioni del codice cortese-feudale, quanto dire a Chrétien stesso, con cui si stabilisce che lo sfruttamento del lavoro risulta grave infrazione ai canoni della courtoisie: perché inflitto a persone deboli e inermi, e perché, in quanto lavoro manuale (in questo caso, la tessitura), riservato ad altre classi — le classi inferiori- di popolazione, che comporta regole di applicazione improprie rispetto al costume cavalleresco; tanto più, poi, che i crudeli sfruttatori ricevono dalle infelici anche un guadagno, infrazione anch'essa al codice cortese. ${ }^{4}$ Pratica, dunque, quella della tessitura, che reca onta e vergogna.

Con questa invenzione, Chrétien riesce a dar forma ad un'emblematica rappresentazione di forte valenza anti-cortese, valevole per essere reimpiegata in situazioni analoghe, ed affrontare un uso paradigmatico di prova avventurosa tra le più temibili. Non è allora conseguenza inattesa che la situazione si condensi in elemento topico, sorta di motivo narrativo valido al pari di altri motivi romanzeschi radicati invece in qualche fondo arcaico (lotte contro avversari soprannaturali, magie), e che lo si ritrovi ripreso e funzionalizzato in altri testi. Con un'importante modificazione, però: poiché vi vengono commutati sesso e statuto dei personaggi colpiti dalla condanna, i quali divengono questa volta cavalieri: dizione in cui è implicito che si tratti di 1) esseri umani dotati di una forza fisica maggiore di quella posseduta dalle povere puceles; e di 2) incaricati di una missione etica.

Dopo l'Yvain, in effetti, eventuali attestazioni di questo episodio-motivo si rinvengono nei romanzi arturiani successivi. L'applicazione, è vero, non è troppo frequente. Un'indagine su un gruppo abbastanza nutrito di romanzi, compresi tra la fine del XII e la metà del XIII secolo (età classica del romanzo arturiano), 5 ne isola poche occorrenze. Una, però, sembra importante. La si legge in un testo in relazione diretta con Chrétien, per quanto non con

\footnotetext{
${ }^{4}$ Non si deve, però, neanche leggere l' episodio come se fosse una rappresentazione delle autentiche condizioni di lavoro di una fabbrica di operaie: Chrétien non adotta un criterio descrittivo realistico, il lavoro vale in rapporto con la sola dimensione cortese.

${ }^{5}$ Ho esaminato i seguenti romanzi: Erec, Cligès, Lancelot, Yvain, Graal di Chrétien de Troyes; Première Continuation Perceval (o Continuation Gauvain); Continuation Perceval (The Second Continuation, Continuation di Gerbert de Montreuil, di Mannessier); Durmart le Galois; Renaut de Beaujeu, Bel Inconnu, Chevalier à l'épée, Claris et Laris; Guillaume le Clerc, Fergus, Hunbaut, Merveilles de Rigomer, Mule sans frein; Béroul, Tristan, e le due Folies; Raoul de Houdenc, Vengeance Raguidel, Yder.
} 
l'Yvain, bensì con il Conte du Graal; vale a dire nella prima delle Continuations prodotte a complemento dell'ultimo romanzo di Chrétien, continuazione nota anche con il nome di Continuation Gauvain; l'occorrenza è ripetuta in tutte le versioni del testo (version courte, mixte, longue), già del tutto formata a partire da quella che è forse la redazione più prossima all'archetipo, la cosiddetta versione del ms. L (Roach, The First Continuation).

Nella branche VI, sezione finale della Continuation, si contempla l'avventura cui Guerrehés, cognato di Gauvain, deve sottostare, a seguito di una maledizione-predizione che è stata annunciata ad Artù, secondo la quale il cavaliere sarà costretto a coprirsi d' infamia a causa di determinati avvenimenti. L'avventura conduce Guerrehés in un castello deserto, dove, in un giardino, un nano lo affronta e gli espone le condizioni in base alle quali potrà evitare di sostenere il duello con il genius loci, un Cavaliere faé, il Petit Chevalier. Le condizioni sono le seguenti:

Li cevalier que je conquier

Sunt asis au plus vil mestier,

Certes, qui soit en tot lo mont.

Por voir vos di que tisier sont,

Ne ja puis n'en ierent osté

Par nul home de mere ne,

Ains tisent pailes et bofus

Et dras de soie a or batus,

Si font trop rices pavellons,

Par foi, de diverses façons.

Molt en a grant rente me sire.

(The First Continuation, 8893-8903)

Questa dovrebbe essere la prima testimonianza del motivo in chiave modificata rispetto a Chrétien: comparando i due passaggi, si notano alcune simmetrie intertestuali tra Yvain e Continuation, relative alla natura delle stoffe tessute, all'ineluttabilità della schiavitù, alla mancanza di cibo e di abiti, e al guadagno che ne ricavano coloro che sfruttano l'opera degli infelici:

Yvain

Vit puceles jusqu'a trois cenz

Qui diverses oevres feisoient:

De fil d'or et de soie ovroient...

(5196-5198)

Ja mes n'avrons rien qui nos pleise

\section{Continuation}

Sunt asis au plus vil mestier, Certes, qui soit en tot lo mont.

Por voir vos di que tisier sont

Ne ja puis n'en ierent osté 
Mes mout di ore grant enfance

Qui paroil de la delivrance

Que ja mes de ceanz n'istrons;

Toz jorz dras de soie tistrons...

(5296-5300)

Et de ce ne poons nos pas

Assez avoir viande et dras

Car qui gaaigne la semainne

Vint solz n'est mie fors de painne...

(5312-5315)

110 Et nos somes ci an poverte,

S'est riches de nostre desserte

Cil por cui nos nos traveillons...

(5319-5321)
Par nul home de mere ne,

Ains tisent pailes et bofus

Et dras de soie a or batus,

Molt en a grant rente me sire

La variazione della Continuation è simile nella sottolineatura dell'insoddisfazione delle necessità elementari di nutrimento e vestiario (...ne poons nos pas /Assez avoir viande et dras), anche se ignora del tutto la componente della produzione dell'uno e dell'altro. Essa, tuttavia, reimposta completamente Chrétien: l'umiliazione non deriva da un atto di forza imposto a donne, che sono deboli, di condizione signorile, e di abitudini cortesi, ma si appunta sullo statuto feudale e guerresco dei cavalieri; l'imposizione del lavoro, penoso perché obbligato, si accompagna alla vergogna, poiché viene a concernere l'orgoglio cavalleresco, aggravato dal prepotere della sfida condotta da un personaggio estraneo alla sfera cortese, come è, nella Continuation, il nano. Si è cioè passati dalla negazione della cortesia rivolta alla sfera muliebre, alla deviazione rispetto al codice cavalleresco, e all'irrisione della forza maschile.

Nel raggio dei testi romanzeschi oitanici prossimi cronologicamente non sembrano esservi altre occorrenze comparabili: stando ai romanzi investigati (che pur coprono uno spettro non troppo ristretto), l'occasione testuale più prossima all'Yvain rinvia alla Continuation Gauvain. Si può allora pensare che a questa si debba ricondurre anche l'ipo-testo diretto del passo di Jaufre, che non dovrebbe esserne troppo lontano per cronologia; ${ }^{6}$ ed anche ritenere

${ }^{6}$ La Première Continuation Perceval, nella versione $L$, è datata dai due specialisti Pierre Gallais e Guy Vial all'ultimo decennio del XII secolo (Gallais, L'imaginaire d'un romancier, vol. I, 18ss., e Vial, Le Conte du Graal, 25). Ma anche i rifacimenti delle cosiddette versioni longue 
che la medesima stia alla base di un'occorrenza analoga, che si trova in un testo affine a Jaufre per argomento, ma, questa volta, abbastanza distante per area (piccarda) e per epoca (metà del XIII sec.) di composizione, e dunque presumibilmente ad esso ignoto, le Merveilles de Rigomer: romanzo, per inciso, che anche altrove sembra aver subito l'influsso dalla Continuation: ${ }^{7}$

Mais laiens en ot mout de pris

Qui a vilain mestier sont mis.

Laiens faisoient les ovrages

Dont jou vos vaurai faire sages.

Des qu'al mestier estoient mis,

En un seul jour furent apris

Ne onques nul jour s'en isoient.

$\mathrm{Li}$ vaillant chevalier tisoient

Les siglatons et les cendaus

Et les pailles imperiaus

(Les Merveilles de Rigomer, 6349-6358)

In tutti e tre i casi - Continuation, Jaufre, Rigomer - viene attivata una valenza antropologico-culturale di elevata significazione, presente, ma ancora debole in Chrétien, che fa perno sull'inadeguatezza della mansione e la non-proprietà degli attanti: lavoro improprio e degradante, imposto a dame e a cavalieri. Ciascuna delle tre occorrenze attiva poi ulteriori sollecitazioni. Per Continuation, e per Rigomer, interviene un contesto degradante accessorio, che è, per Continuation, la sfida lanciata dal nano, per Rigomer la localizzazione straniante del castello di Rigomer, sul quale pesa un incantesimo anti-cavalleresco; Rigomer coopta inoltre alla tessitura un elemento ulteriormente abbassante, il lavoro di cucina, al quale è sottomesso l'eroe Lancelot, che patisce un'umiliazione più cocente degli altri cavalieri incantati che popolano il castello, dediti ad occupazioni pesanti ma 'pulite', come tessitura, cura dell'orto, ecc. (6327-6340): ${ }^{8}$ si potrebbe quindi indurne che, nel caso, il lavoro coinvolga, nel romanzo, un elemento di schietta portata culturale come lo sporco e l'impurità (il rinvio è al noto saggio di Douglas, Purity and Danger).

e mixte dovrebbero essere stati scritti intorno alla fine del secolo, benché i manoscritti appartengano a tutto il secolo seguente.

${ }^{7} \mathrm{Vi}$ sono, in effeti, diverse affinità tra i due romanzi: la presenza del cavaliere defunto trafitto dal troncone di lancia (Foerster ed., Merveilles, 1233-1456), Gauvain cavaliere legato al corso del sole, il divieto di mangiare prima dell'avventura, ecc. (Lecco, "Mais l'aventure revient ja").

${ }^{8}$ Su questo aspetto, vid. Trachsler (Lancelot aux fourneaux). 
Jaufre carica anch'esso valenze di rilevante potere antropologico, ma giocate su un altro versante ancora: l'incompatibilità con gli usi della società civilizzata, che prevedono determinate modalità di trasformazione e manipolazione delle materie alimentari e tessili, in relazione al nutrimento e all'abbigliamento: dunque, la prova cui dovrebbe sottostare Jaufre pertiene all'arte particolare che conduce alla produzione dei manufatti, che è estranea ai cavalieri, ma anche alla concreta possibilità di essere espulso dal contesto delle regole comportamentali che regolano la civile condizione umana. ${ }^{9}$ La gravità della sfida del Cavaliere della Blanca Lança risiede nell'esclusione dalla civiltà cui andrebbe incontro Jaufre se cedesse alla tentazione della rinuncia che sembra sfiorarlo per un istante. Ed è tanta la disperazione che Jaufre intravvede nel possibile cedimento, da spingerlo istantaneamente ad accettare un combattimento al quale, come s'intuisce, ${ }^{10}$ rinuncerebbe volentieri.

\section{SEGNI NEL TESTO}

In Jaufre l'episodio del lavoro di tessitura è profondamente radicato entro un codice di significati e di scrittura, che trova la debita interpretazione a contatto con un sistema di segni saldamente strutturato. ${ }^{11}$ Opera scritta con manifeste finalità d'encomio ad un re d'Aragona (Lee, 12-15), ${ }^{12}$ il romanzo ottempera a questa finalità attraverso una serie di espedienti narrativi e retorici (la distribuzione delle aventures iniziale e finale di re Artù, le molteplici e graduate sfide sostenute da Jaufre), che, dal testo, proiettano sulla figura del 'vero' re d’Aragona le implicazioni fittiziamente sostenute da Artù: il valore attribuito ad Artù sottende l'esaltazione del re autentico. Di questi espedienti, il più sottile e ingegnoso consiste nella costruzione di una 'semiosfera' (per questo concetto, Lotman, La semiosfera): di una serie di elementi interpretabili segnicamente, di segni 'orientati', inseriti come programmatico rinvio e guida all'interpretazione, tanto inter-relati da potersi dire costituiti in sistema, con

\footnotetext{
${ }^{9}$ La proibizione di cibarse di pane e bere vino, ad esempio, sembra sottintendere l'imposizione di consumare solo cibi crudi, non preparati ( vid. naturalmente, Lévi-Strauss, Le cru et le cuit).

${ }^{10}$ Come sembrano lasciar intendere i vv.1475-1477 (Lee, 106).

${ }^{11}$ Ho trattato estesamente questo aspetto nel saggio "Artù e l'incantatore". Per un'interpretazione semiotica del Jaufre si veda anche l'importante studio di Jung, "Lecture de Jaufré".

${ }^{12}$ I possibili candidate vanno da Alfonso II d’Aragona (1162-1196), a Pietro II (11961213), a Jaime I (1213-1276). Se il romanzo può essere datato, da elementi interni (rimandi testuali, stilistici, ecc.), al 1225-1230 c., il dedicatario sarà forse da identificare in quest'ultimo.
} 
valore ideale ed ideologico insieme. Questi segni coprono almeno tre settori tematici (che, procedendo ad uno stesso scopo, si completano reciprocamente, sovente sovrapponendosi, così da trarre significazione dal reciproco interagire): uno fa capo al nutrimento, un secondo all'abbigliamento, un terzo al dono e al donare, nucleo che possiede una valenza di maggiore portata, in cui si risolvono e riassumono i precedenti (Lecco, “Artù e l'incantatore", 14-24).

Tutti, come si vede, nella loro diversa tipologia, sono in relazione con un aspetto fondamentale dell'esistere e del comportamento umano, la necessità della sussistenza, intesa in base a norme culturali impostate da secoli, cui si sommano le peculiari regole della vita feudale-cortese: la loro presenza si spiega correttamente appunto mediante l'uso di categorie antropologiche, per la cui analisi è opportuno spingersi in questa direzione. Dei tre nuclei, l'ambito del nutrimento sembra coprire meno occorrenze, essere meno esteso. In diverse occasioni, esso risponde solamente agli svolgimenti della narrazione, e non possiede dunque un particolare valore di segno: si vedano le varie descrizioni di pranzi e di cene (ad es. 508ss, 8201ss, 9225ss, ecc.), segnicamente indeterminate, per quanto efficaci nel delineare l'atmosfera di fasto della corte arturiana. Le situazioni connesse al nutrimento diventano invece degne di considerazione in tre casi: nell' iniziale episodio che vede Artù, i suoi cavalieri e cortigiani non potersi mettere a tavola, perché nessuna avventura è ancora sopravvenuta a corte (140-161); nell'episodio dell'incontro di Jaufre con il boer (4201) che sta portando cibi alla corte di Monbrun (4202ss.), e che, interrogato dal cavaliere sulla natura dei lamenti che si odono a Monbrun, erompe in gesti furiosi che lo portano a distruggere i beni in consegna; nella mancanza di cibo che caratterizza la vicenda dell'infelice re Melian, la cui dimora di tormento (4818ss.), una terre gaste, appare in netto contrasto con la casa colma di abbondanza di Augier e dei suoi figli che la precede nel romanzo.

Anche le occorrenze collegate all'abbigliamento rispondono all'esigenza di provvedere a necessità vitali, codificate in base a norme stabilmente costituite, che devono assicurare la copertura e la protezione dalla nudità ad esseri umani che sono, nel contempo, appartenenti alla corte, quindi, per metonimia, all'intero regno. Il nucleo-tema del vestiario è molto esteso nel Jaufre, secondo una dialettica di distruzione-restituzione. Nell'episodio di apertura del romanzo, Artù, che ha assunto su di sé l'onere del rinvenimento di un'avventura (condizione da cui dipende il buon andamento della corte), si trova a dover fronteggiare una situazione di perdita di abiti: afferrato dalla bestia e sospeso nel vuoto del precipizio, il re viene infatti salvato da «Galvan»e degli altri cavalieri, che ammucchiano i propri abiti nel punto dove il re debe lasciarsi cadere:

Medievalia 50, 2018, pp. 105-122 


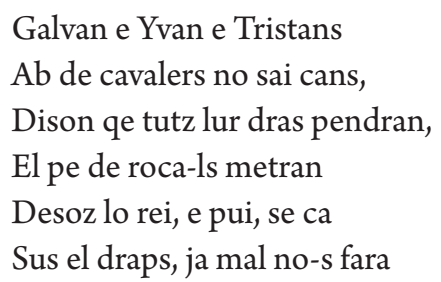

(387-392)

Come quella alimentare, la carenza di vestiario rientra nella situazione di mancanza che pervade tutto il Jaufre, alla quale cerca di ovviare Artù, che si espone in prima persona alla ricerca dell'avventura, ai pericoli che essa comporta, e che, d'altra parte, come veniamo a sapere dai vv. 446-458, ha combinato appositamente tutta la faccenda mediante un celato accordo con uno dei suoi cavalieri, dotato dell'abilità magica di trasformarsi: accordo che, una volta espletato, rende il cavaliere-enchanteur degno di un ricco dono. La metamorfosi del cavaliere si ripete verso la fine del romanzo, quando Jaufre, con le prove del suo valore cavalleresco, ha reso ormai inutile il pur nobile trucco (9911-10062). Sino a quel momento, gli appartenenti alla corte arturiana e quelli di Monbrun vedono, in varie occasioni, distruggere i propri abiti: li perdono, li stracciano nei momenti di dolore o di paura, ad esempio quando, al momento della seconda metamorfosi, vedono Artù affrontare il falco sotto le cui spoglie si nasconde il cavaliereincantatore, e si lasciano andare al dolore per la presunta perdita del re:

\author{
E son s'en el palais entrat, \\ On an lo bon rei atrobat, \\ San e sals e sen encombrier. \\ E fon ab aquel cavallier \\ Que-ls a-l jorn aissi encantatz \\ E-ls fa totz anar esquintatz
}

L'avventura del falco rapitore è tuttavia l'ultima cui Artù e la corte debbano sottomettersi. Venute meno le condizioni della metamorfosi, e dell'avventura da dover sostenere in prima persona, in corrispondenza con la ritrovata dignità, il re decide, con deliziosa magnificenza e come atto di riparazione, di far confezionare e donare a ciascuno, cavaliere, cortigiano o giullare, un nuovo abito. Egli convoca tessitori e sarti, e persino concede che ognuno possa scegliere la stoffa che più gli piace: 
E aqui mezeis fa cridar

Le reis, que qui vol far taillar

Vestirs, qu'el venga-ls draps chaussir,

Ez apres fara-ls hom cosir.

E que-us iria alre ditzen?

Que non feron nul'altra ren

Tuitz li sartor ne-l costoriers,

Mas vestirs far als cavalliers

$\mathrm{E}$ a las domnas eissamentz.

(10111-10119)

Con la distruzione degli incantesimi, ed il restaurato buon governo, nel regno arturiano ritorna la ricchezza: ricchezza e abbondanza, come rinnovata immissione e circolazione dei beni e del lusso che si addicono ad un re, le quali riportano la restaurazione dell'abbigliamento, e vengono poi siglate da un'ulteriore donazione, costituita da manufatti d'oro e d'argento:

Ja non sera mais veramenz
A neguna cort tant taillat,
Tantz bon pali ne tant cendat,
Ne dat tan d'aur ni tant d'argen,
Ne tant bel arneis eissamen

(10120-10124)

L'inserimento dei nuclei-temi alimentare e vestiario, hanno funzione di chiarire il ruolo del re, che (come per il re d'Aragona) è provvidenziale, paterno e riparatore: e si esplica dunque giustamente attraverso il dono. Il donare, e i doni, come terzo nucleo tematico-semiotico, sono il mezzo mediante cui Artù può intervenire sulle carenze che si vengono a produrre nel suo regno, e sono, d'altronde, il mezzo per ricostituire l'integrità e la giusta funzione che la figura regale deve coprire all'interno del regno. Come longa manus, mano materiale, fisica, del re, Jaufre sostiene le avventure che il re, in quanto re e non cavaliere, può sostenere solo fittiziamente; ma è poi appunto ad Artù che pertiene l'atto finale: momento di ricostituzione, di restituzione, che Artù sostiene proprio quando, terminate le avventure e distrutti tutti i nemici (Taulat de Rogemont, Felon d'Albarua), può restituire a tutti gli abiti, con il taglio, la cucitura, e, prima ancora, la tessitura degli abiti, che ciascuno degli appartenenti alla corte è invitato a scegliere. 
Al centro della fenomenologia dei codici vestimentari-tessili sta, nel Jaufre, un'importante valorizzazione: la mancanza degli abiti (più ancora che del cibo) è in relazione con una mancanza di sicurezza sociale, quanto dire, nel mondo feudale, con l'infrazione del rispetto delle regole di protezione e cura dei sudditi da parte del sovrano. La minaccia del Cavaliere della Blanca Lança investe, attraverso Jaufre, l'eventualità di questa infrazione. Tessitura, e confezione degli abiti, sottratte a chi ne ha il compito e la competenza, ed imposte a chi non ne conosce le modalità ed è inabile a provvedere, costituiscono, prima ancora che una prepotenza, la rovina delle norme di civiltà, doppiata sul versante umano e su quello feudale, dato che l'attività imposta a Jaufre è impropria per lui come soggetto civile e come cavaliere feudale. Il topos dell'imposizione della tessitura, originato da Chrétien, rivela quindi, in

116 Jaufre, una notevole adattabilità e proprietà a darsi, nella letteratura romanzesca, come motivo d'impiego costante per esprimere il disagio dell'uscita, e/o minaccia di uscita, dal sistema dei valori cavallereschi.

\section{Jaufre e la Continuation Perceval}

Il riscontro del topos motivale pone ancora un quesito. Quali possono essere stati, se vi sono stati, i rapporti tra Jaufre e Première Continuation Perceval. La domanda si è imposta da tempo, poiché scaturita in relazione ad un problema ecdotico di Jaufre, la lezione offerta da uno dei due mss. completi del romanzo, il ms. Paris B. N. fr. 2164 (conosciuto dagli editori come A; per questo problema vid., da ultimo e riassuntivo, Lee, nota al v. 6654). Al v. 6654 di questo ms. si legge un riferimento al siniscalco Keu, che viene colpito dal cavaliere Taulat $a b$ lo paon, cioè con una coscia di pavone adoperato come arma: questa lezione contrasta con quella dell'altro ms. completo, il Paris B. N. fr. 12571 (ms. B), dove il testo trascribe un più comune baston. La lectio difficilior, «paon», viene attualmente considerata più credibile da editori e commentatori, i quali la fanno discendere appunto da un episodio della Continuation, contenuta nella branche IV (version courte L), Chastel Orguelleus, vv. 3655-3788ss., sp. vv. 3767-3780 (Première Continuation Perceval).

La presenza del motivo della tessitura, che si può pensare desunto, ed in modo esclusivo, dalla Continuation, potrebbe intervenire positivamente in questo senso, suffragando con una prova testuale la possibilità che la lezione del ms. sia di natura autoriale, appartenente alla versione d'origine. Potrebbe dunque trattarsi di un buon argomento per accertare l'eventualità di una conoscenza della Continuation da parte di Jaufre, in grado di computare 
definitivamente il testo oitanico tra le fonti del testo scritto in provenzale. La questione non è di facilissima soluzione, perché prove ulteriori non sono assolutamente certificanti. Esiste forse qualche altro indizio suscettibile di indirizzare l'inchiesta in questa direzione. Qualcuno, forse, con qualche possibilità in più, ma non certezza. Come il motivo, che è folclorico ed insieme strutturale, dell'impedimento a mangiare prima che sia giunta a corte un'avventura, quello che l'Index des Motifs narratifs di A. Guerreau-Jalabert repertoria come: C 231 Tabu: eating before certain time (31). La Continuation ne reca esempi, ai vv. 2215-2239 e 3123-3139 (Première Continuation Perceval, versione $L$, t. III, 140 e 196), che avrebbero forse potuto essere utilizzati da Jaufre, per quanto anche diversi altri romanzi, composti all'incirca all'altezza cronologica di Jaufre, ne rechino traccia: ma è pur vero che, del tabu, si trova una precoce realizzazione nel Conte du Graal, vv. 2819-2829 (Poirion et al. ed., Chrétien de Troyes, Euvres complètes, 755).

In altri casi, la possibilità, non escludibile, resta tuttavia molto incerta. Come per la scena iniziale della branche V, dove un cavaliere sconosciuto offende la regina Guenevere ( $L, 6765-6842)$, nonostante l'intromissione di Keu, che potrebbe far pensare al 'secondo' prologo di Jaufre, che inizia con l'arrivo di Jaufre, dopo l'episodio d'apertura dove Artù deve sostenere in prima persona l'avventura (Continuation, 439-442; Jaufre, 585ss.); o ancora per la 'vergogna del giardino' cui Jaufre va incontro quando si addormenta nel giardino di Monbrun (3181ss.) e che potrebbe rammentare un particolare dell'episodio di Guerrehés sopra citato (Continuation, 562; Jaufre, 153); od ancora, persino la descrizione di Felon d'Albarua, dal viso mostruoso (87798797), che potrebbe ricordare la caratterizzazione del nano cort, bocu et desfet, che Gauvain incontra nella I branche (Continuation, 2536-2571 —questo passo si legge nei mss EU, in Continuation II, 76-77-; Jaufre, 314). Ma non è dato spingersi troppo oltre: le eventuali affinità potrebbero sempre coinvolgere un'iniziativa 'a monte', pregressa, da identificare in Chrétien: la caratterizzazione, ad esempio, del nano dai tratti bestiali potrebbe dipendere meno dall'analoga figura della Continuation che dal mostruoso custode dei tori dell'Yvain, peraltro molto famosa. L'uso del motivo della tessitura (e, con cautela, quello dell'interdizione cibaria) va inteso come tassello che può indicare una possibile conoscenza della Continuation, da parte dell'Autore del Jaufre: in tal senso certificando anche la plausibilità della lezione paon come più esatta per l'apparato di $A$. Se, poi, questa conoscenza sia stata estesa a tutto il testo o limitata ad alcune parti, se sia stata di prima mano o di seconda, diretta (ottenuta per lettura) o indiretta (per ascolto), non è dato stabilire con certezza. 


\section{COMmistione DEL MOTIVO}

Piuttosto, si può provvisoriamente chiudere il discorso (provvisoriamente, perché non si esclude di poter rinvenire in futuro qualche ulteriore testimonianza del motivo in sede oitanica) ${ }^{13}$ con un'ultima attestazione della condanna al lavoro come motivo d'uso tra i materiali di costruzione della scrittura romanzesca.

In causa, nel caso, va chiamato il romanzo di Claris et Laris, testo del pieno XIII secolo, che esula dall'area cronologica e, forse, culturale di Jaufre (Alton, Claris et Laris). Non vi si ha a che fare con la tessitura, ma con un'occupazione di schietto sfruttamento fisico: i cavalieri, prigionieri del consueto reame fatato visitato dai due protagonisti, sono costretti a trasportare pietre:

ii. autres varlez les menerent

par les degrez en une sale;

Maint chevalier a coulour pale

Ont en cele sale veu,

Mainte besoingne orent eu.

Touz les jours du mont laboroient,

Pierres pesanz et granz portoient,

Tant n'avoient le jor porte,

La nuit n'eussent reporte.

Dui glouton, ce sachiez, les gardent,

Qui de granz courgiees les chargent,

Souvente foiz par les costez;

Si vous di qu'il y a de tez

Qui ont les hanches pertuisiees

Et les espaules depeciees

Des faiz soustenir et porter;

Petit se pueent deporter

Cil, qui vivent en tel mesaise,

Car il n'ont chose qui lor plaise;

$\mathrm{Ne}$ menjuent fors iave et pain

De quoi il esbatent lor fain;

Nepurquant chevalier estoient

Et maint biau coup feru avoient.

(Claris et Laris, 2346-2368)

${ }^{13} \mathrm{E}$ in altre letterature ancora. 
Nnostante la variazione della pena, si riconoscono agevolmente i tramiti testuali da cui il passo scaturisce. La fonte diretta sembra essere Chrétien, essendo comparabili tra loro più sequenze:

1) i/le operai/operaie hanno corpi feriti e vesti strappate:

Yvain

Mes tel povreté i avoit

Que desliées et desceintes

En i ot de povreté meintes,

Et as mameles at as cotes

Estoient lor cotes derotes,

Et les chemises as dos sales

(5200-5205)
Claris

Si vous di qu'ily a de tez

Qui ont les hanches pertuisiees

Et les espaules depeciees

Des faiz soustenir et porter...

(2358-2361)

2) la gravosa fatica li rende pallidi e smunti:

Les cos gresles et les vis pales

De fain et de meseise avoient

(5206-5207)
Maint chevalier a coulour pale

Ont en cele sale veu,

Touz les jours du mont laboroient,

Pierres pesanz et granz portoient...

(2348-2351)

3) il cibo è ridotto, consiste in poco pane ed acqua:

Toz jorz serons povres et nues,

Cil, qui vivent en tel mesaise,

Et toz jorz fain et soif avrons;

Car il n'ont chose qui lor plaise;

Ja tant chevir ne nos savrons

Que mialz en aiens a mangier.

Ne menjuent fors iave et pain

Del pain avons a grant dangier,

De quoi il esbatent lor fain;

Au main petit, et au soir mains.

(2363-2366)

(5302-5307)

4) l'imposizione della schiavitù è dovuta a due glouton, paritari, per costituzione e viltà (e per assonanza) ai due luiton dell'Yvain:

...en cest chastel...

Ou il a deus filz de deable,

Dui glouton, ce sachiez, les gardent

$[\ldots]$

Que de fame et de netun furent...

(5272-5275)

A differire sono invece il sesso dei ridotti in schiavitù, che in Claris et Laris sono uomini, e la loro posizione sociale: si tratta di cavalieri, e questa indicazione, 
memoria di un passato che nel romanzo continua, nonostante tutto, a connotare i cavalieri, e li connota così per sempre, è indice di una coscienza di classe, che si apre e confonde con la più nobile dignità umana. In questa sostituzione, $\mathrm{Cla}$ ris denuncia la conoscenza della Continuation: primo, e più diffuso, esempio di trasformazione in senso caballeresco e feudale del motivo, che funziona come archetipo (o co-archetipo, con l'Yvain) ispiratore di tutta una tradizione.

Nel novero dei motivi romanzeschi, quello del lavoro imposto (tessitura o altro), non ha un'origine folclorica, e dunque dotata di nobili ascendenze mitologiche, non dipende da antichi lasciti di culture arcaiche, diffusi nel tempo, e più o meno stilizzati e cristallizzati dalla cultura medievale. Ricopre invece una forte valenza culturale tutta contemporanea, ${ }^{14}$ che la stilizzazione letteraria compatta in espressione formalizzata, d'esemplare senso 120 etico collegato alla contemporaneità della società medievale. Parla anche, tra l'altro, della complessità della costruzione, e del significato, dei motivi narrativi della letteratura medievale, che non sono da interpretare e intendere tutti sullo stesso piano, né si formano tutti nello stesso modo, e che sono in uso ora come soli materiali d'intreccio, ora anche come elementi strutturanti, da cui dipende la distribuzione del racconto.

\section{BIBLIOGRAFIA}

Alton, Johann (ed.), Li Romans de Claris et Laris, Amsterdam: Rodopi, 1966.

Breuer, Hermann (ed.), Jaufre. Ein Altprovenz. Abenteuerroman des XIII. Jahrhunderts, Göttingen: Niemeyer, 1925.

Brunel, Clovis (ed.), Jaufre. Roman arthurien du XIII e siècle en vers provençaux, Paris: Société des Anciens textes français, 1943.

Foerster, Wendelin (ed.), Les Merveilles de Rigomer von Jehan. Altfranz. Artusroman des XIII. Jahrhunderts, t. I, Dresden: Niemeyer, 1908.

Douglas, Mary, Purezza e pericolo. Un'analisi dei concetti di contaminazione e tabu, Bologna: Il Mulino, 2003 (Purity and Danger, Harmosaworth: Penguin Books, 1970).

Gallais, PIERre, L'imaginaire d'un romancier français de la fin du XIIe siècle. Déscription raisonnée, comparée et commentée de la Continuation Gauvain, 4 voll, Amsterdam: Rodopi, 1988-1989.

${ }^{14}$ Un esempio analogo, che ha del resto numerosi punti di contatto, se non di sovrapposizione, con questa proposizione culturale, si ha nel lavoro di Le Goff, Osservazioni, che esamina il funzionamento di codici semiotici particolarmente evidente nel romanzo di Chrétien de Troyes Erec et Enide. 
Guerreau-Jalabert, Anita, Index des Motifs narratifs dans les Romans Arthuriens français en vers (XIIe-XIIIe siècles), Genève: Droz, 1992.

Jung, Marc René, "Lecture de Jaufré", in Mélanges offerts à Carl Theodor Gossen, t. I, Berne-Liège; Krancke, 1976, 427-451.

LAfont, Robert, "Relecture de Cabra juglar", Revue des Langues Romanes, 105, 2000, 337-377.

Lavaud, René e René Nelli, Le Roman de Jaufre, in Les Troubadours, t. I, Bourges: Desclée de Brouwer, 1960, 621-1021.

Lecco, Margherita, "Artù e l'incantatore. Corrispondenze strutturali e semantiche nel Jaufré, in EAD., Saggi sul Romanzo del XIII secolo, Alessandria [Italia]: Edizioni dell'Orso, 2003, 3-30.

Lecco, MArgherita, “Mais l'aventure revient ja. Retaggio «folclorico» e scrittura nelle Merveilles de Rigomer", in EAD, Saggi sul Romanzo del Xiı secolo, Alessandria [Italia]: Edizioni dell'Orso, 2003, 55-69.

Lecco, Margherita, “Testo, immagine (e un intertesto sconosciuto?) nel ms. B. N. fr. 2164 del Roman de Jaufre", in Materiali arturiani nelle Letterature di Provenza, Spagna, Italia, a cura di M. L. (saggi di Berthelot, Gouiran, Lazzerini, Lecco, Lee, Orazi, Ueltschi, Wahlen), Alessandria: Edizioni dell’Orso, 2006, 73-95.

Lee, Charmaine (ed.), Jaufre, Roma: Carocci, 2006.

Le GofF, JaCQUes, “Osservazioni sui codici di abbigliamento e alimentari nell’ Erec et Enide”, in Il meraviglioso e il quotidiano nell'Occidente Medievale, Bari: Laterza, 1999, 138-147.

Levi-Strauss, Claude, Mythologiques 1. Le cru et le cuit, Paris: Plon, 1964.

Limentani, Alberto, "I problemi del Jaufre, l'umorismo, e una contraffazione del Conte du Graal”, in L'eccezione narrativa. La Provenza medievale e l'arte del racconto, Torino: Einaudi, 1977, 78-101.

Lotman, JuRij M., La Semiosfera. L'asimmetria e il dialogo nelle strutture pensanti, a cura di S. Salvestroni, Venezia: Marsilio, 1985.

Pirot, Francois, Recherches sur les connaissances litteraires des troubadours occitans et catalans des XIIe et XIIIe siècles, Barcelona: Real Academia de Buenas Letras, 1972.

Poirion, Daniel et al. (eds.), Chrétien de Troyes, OEuvres complètes, édition publiée sous la direction de Daniel Poirion, Paris: Gallimard, 1994.

Pontecorvo, Aurelia, "Una fonte del Jaufre", Archivum Romanicum, 22, 1938, 399-401.

Roach, William (ed.), The Continuations of the Old French Perceval of Chrétien de Troyes, Philadelphia: The American Philosophical Society, 1952, vol. III, The First Continuation (mss. ALPRS).

Ross, Gilbert A., Jaufre. An Occitan Arthurian Romance, New York-London: Garland, 1992.

Medievalia 50, 2018, pp. 105-122 
Trachsler, Richard, "Lancelot aux fourneaux. Des éléments de parodie dans Les Merveilles de Rigomer", Vox Romanica, 52, 1993, 80-93.

Vial, Guy, Le Conte du Graal, sens et unité. La Première Continuation, Genève: Droz, 1987.

122 